\title{
Early Social Interaction: A Case Comparison of Developmental Pragmatics and Psychoanalytic Theory Michael A. Forrester (Cambridge University Press, 2015)
}

\author{
Karin Osvaldsson \\ Linköping University, Sweden \\ karin.osvaldsson@liu.se
}

It was with great enthusiasm, but also, I must confess, some hesitation I took on the task of reviewing this book. Being a psychoanalytically trained child psychologist turned ethnomethodologist myself, I may be seen as either the perfect or the worst reviewer of this kind of enterprise.

Forrester sets out to investigate how a child learns how to talk and through this process, how the child becomes languaged and part of a culture. It is a single case-study, where we follow the development of Ella. The study is longitudinal, spanning over several years; from Ella's earliest utterances, to the display of more and more elaborate interaction and language skills. The carefully chosen examples are transcribed using Jeffersonian conventions and every example is accompanied by a link to where the actual recording itself may be viewed.

One part of this investigation has to do with the child learning how to understand and interact with people and the surrounding world. This part is labelled the social-action or doing part by Forrester, and in order to investigate this he adopts ethnomethodology, well equipped to take on the task. But his study also has another aim. It wishes to interpret a child's recognition of feeling or affect, and here is where he chooses a psychoanalytical framework to help his interpretations. 
This is no easy task. Ethnomethodology (EM) and conversation analysis (CA) has taken as its prime aim to re-specify, among others, psychological phenomena as participant's concerns, while psychoanalysis is concerned with the inner emotional life of humans. This makes the two perspectives fundamentally different and the book does not combine the two into the same analysis. It is rather organized as an alternating analysis of 'outer' as well as 'inner' aspects of Ella's development. This is probably a wise move.

After a comprehensive introduction, Chapters 2 and 3 offer an overview of EM/CA with a specific focus on developmental pragmatics and a child focused conversation analysis. Here I especially appreciate that Forrester recognizes the undervalued work of Mackay (1974) and his EM informed critique of theories of socialization. There is also a useful introduction of Harvey Sacks's work on thinking as not merely individual but social. For Sacks, thinking is among others an observational skill, which he exemplifies with the concept of lying. Learning how to get away with a lie as well as detecting lies in others are seen by Sacks as having to do with learning to 'see' others' thoughts, and are important social skills to be acquired (Sacks, 1980, 1995).

Chapter 4 gives us a brief introduction to the psychoanalytical concepts and theories used for the analysis of emotions and the development of early social relations. The chapter is mostly dedicated to British object-relation theorists such as Melanie Klein and Donald Winnicott. The choice of an object relational framework surprised me a bit, I would have gathered, with Forrester's interest in children's social development that he may rather have chosen more contemporary theorists such as Daniel Stern (2000).

Chapter 5 outlines 'an account of human interaction that emphasises the significance and pervasiveness of both social action and psychological affect' (p.64, original italics). It details the virtues of an EM/CA fine-grained analytical approach to the analysis of social interaction. But Forrester is also critical towards this approach and argues that EM/CA hosts almost an obsession with order. This he claims, causes EM/CA to miss out on the analysis of interaction breakdown. Billig (1999) has argued that Freud's concept of repression, in interactional terms could be equivalent to the learning of what (not) to say. Forrester extends Billig's argument when he discusses interactional moments where the participants are very upset. He presents us with three extracts, where he argues that the participants' inner emotions have become so strong they can no longer be repressed. In his view, this is the reason why emotions manifest in interaction. Forrester

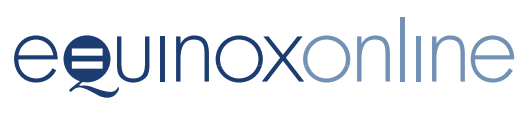


proposes that the extracts represent moments where the CA principle of "order at all points" breaks down. Here, the analysis is less than convincing. It seems to me that Forrester holds a slight misconception of what the EM/ CA concept of order really entails. In my reading, these are extracts where affect and emotions are manifest in the interaction, but where interaction as such, in terms of participant orientation has not broken down.

Chapter 6 presents us with research practice and methodology. We are also informed that the target child, Ella, is the author's own daughter. To perform and publish research that include close relatives is a delicate matter. This is only discussed briefly but I would have liked it to see a more developed ethical discussion on these matters. The dual ambition to perform an analysis that includes both Ella's inner (psychoanalysis) and outer (EM/CA) worlds is also further developed.

Chapters 7-12 are alternatively dedicated to the analysis of the development of extrinsic (social action) versus intrinsic (emotion) competence and recognition. The reader is presented with analyses of the emergence of self-repair skills (Chapter 7), how to repress or the learning of what (not) to say (Chapter 8), and the development of a competence to formulate and answer questions (Chapter 9).

In Chapter 10 the focus is again on emotions. Forrester makes use of Winnicott's concept of the 'transitional space'. The chapter analyses instances of play as manifestations of Ella's separation-individuation process but also the emerging ability to do disagreement.

The last two analytical chapters are dedicated to the concept of self and how that may be analysed as a way of positioning yourself in the world. In Chapter 11 Forrester makes fine use of membership categorization analysis in order to help us see how Ella gradually displays more and more sophisticated ways of self-positioning. Chapter 12 instead makes use of psychoanalysis for an analysis of Ella's development of a sense of self.

Chapter 13 summarizes the argument that several forms of analysis are needed for a fuller narrative of the process of enculturation in terms of becoming languaged. And it has to be said, I am very intrigued by the ambition behind this book. It is indeed very true that EM is unable to answer a whole range of questions concerning the origins and reasons for human affects. On the other hand, EM is very good at picking up on the recipient design of affective displays and may very well answer why people do the things they do in interaction. Personally, I am a bit hesitant to the sharp division of the inner versus the outer worlds that Forrester's

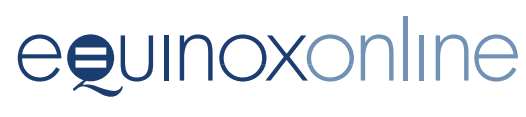


main argument rests upon. I would feel a lot more comfortable with a stricter proof procedure. Throughout the twentieth century psychoanalytic theory has been an important interpretative tool for human drives and inner motives, as it still is today. It is a powerful therapeutic tool for the analysis of personal narratives and personal change. But this is something else than using psychoanalysis for the analysis of in situ social interaction.

Finally, someone like me, being more on the extrinsic side, I am not fully comfortable with the chapters in the book that are theory, rather than empirically driven. I am on the other hand very impressed with the longitudinal study of the development of interactional skills. On these matters this book fills a gap that has not, to my knowledge, been investigated so thoroughly since Ochs and Schieffelin's (1979) and Wootton's (1997) pathbreaking studies.

\section{References}

Billig, M. (1999). Freudian repression: Conversation creating the unconscious. Cambridge: Cambridge University Press.

Mackay R. W. (1974). 'Conceptions of children and models of socialization.' In R. Turner (ed.), Ethnomethodology: selected readings, pp. 180-193. Harmondsworth: Penguin.

Ochs, E. \& Schieffelin, B. B. (eds). (1979). Developmental pragmatics. New York: Academic Press.

Sacks, H. (1980). Button button who's got the button. Sociological Inquiry, 50(3/4), 318.

Sacks, H. (1995). Lectures on conversation, vols I and II. Oxford: Blackwell Publishers.

Stern, D. N. (2000). The interpersonal world of the infant: A view from psychoanalysis and developmental psychology. New York: Basic Books.

Wootton, A. J. (1997). Interaction and the development of mind. Cambridge: Cambridge University Press. 